\title{
Production of zinc oxide nanowires power with precisely defined morphology
}

\author{
Júlia Mičová*, Zdeněk Remešs*,***, Yu-Ying Chang ${ }^{* *, * * *}$
}

\begin{abstract}
The interest about zinc oxide is increasing thanks to its unique chemical and physical properties. Our attention has focused on preparation powder of $1 \mathrm{D}$ nanostructures of $\mathrm{ZnO}$ nanowires with precisely defined morphology include characterization size (length and diameter) and shape controlled in the scanning electron microscopy (SEM). We have compared results of SEM with dynamic light scattering (DLS) technique. We have found out that SEM method gives more accurate results. We have proposed transformation process from $\mathrm{ZnO}$ nanowires on substrates to $\mathrm{ZnO}$ nanowires powder by ultrasound peeling to colloid followed by lyophilization. This method of the mass production of the $\mathrm{ZnO}$ nanowires powder has some advantages: simplicity, cost effective, large-scale and environment friendly.
\end{abstract}

K e y w o r d s: zinc oxide nanowires, hydrothermal growth method, scanning electron microscopy (SEM)

\section{Introduction}

Zinc oxide is important function material for its special physical and chemical properties, such as high chemical resistance, high mechanical and thermal stability. $\mathrm{ZnO}$ as a semiconductor in group II-VI, whose binding capacity is on boundary between covalent and ionic semiconductors. A high refractive index, broad energy band (3.37 eV [1]), large exciton binding energy (60 meV [2]) and the high thermal conductivity are attractive properties with potential for application in optoelectronics [3], electronic devices such as energy generators [4], (gas) sensors [5], converter and laser technology [6]. Another positive aspect of this material is the variety of structures. Examples of one-dimensional (1D) structures are nano-rods [7], -wires [8], -tubes [9], -needles [10], -ribbons [11], -belts. Two-dimensional (2D) structures of zinc oxide can be nano-pellets, -sheet, -plate [12]. Examples of 3D structures of zinc oxide include flower, snowflakes, etc $[13,14]$. Our attention was focused on preparation powder of one-dimensional zinc oxide nanowires. Several methods have been reported for the synthesis $\mathrm{ZnO}$ nanowires, eg thermal evaporation [15], pulsed laser deposition (PLD) [16], chemical vapour deposition (CVD) [17], metal organic CVD [18], electrochemical deposition [19] and template method [20]. However, these techniques require extremely growth conditions (high pressure, temperature and dangerous chemicals) and expensive instruments. But we have used low cost growth technique under mild conditions - the hydrothermal growth method [21]. This method has some advantages. The procedure is environmentally friendly because requires temperature and pressure closely to the living conditions on Earth. The costs for precursors, instrumentation and energy are lower. The essential condition for this method present of seeding layer to achieve growth aligned nanowires with precise morphology. The salt of zinc nitrate hexahydrate is source $\mathrm{Zn}^{2+}$ ions required for building up $\mathrm{ZnO}$ nanowires. The molecules of water provide $\mathrm{O} 2$ - ions. The molecules of water provide $\mathrm{O}^{2-}$ ions. The hexamethylenetetramine (HMTA) as weak base which hydrolyze in the aqueous environment and produce $\mathrm{OH}^{-}$ions. The rate of this reaction is important in the synthesis process. The high $\mathrm{pH}$, which would have little contribution to the $\mathrm{ZnO}$ nanowire oriented growth, and eventually results in fast consumption of the nutrient and prohibits further growth of $\mathrm{ZnO}$ nanowires. The growth process of $\mathrm{ZnO}$ nanowires can be controlled by concentration of precursor, growth temperature and growth time. In general, the growth time and temperature control the morphology of zinc oxide nanowires and the concentration determines the density of nanowires.

\section{Experimental procedures}

The nucleation layer was prepared by magnetron sputtering of the Al-doped $\mathrm{ZnO}$ target doped on soda-lima glass substrates $(25.4 \mathrm{~mm} \times 76.2 \mathrm{~mm}$, after cleaning process by sonication subsequently in acetone, isopropanol and deionized water for $5 \mathrm{~min}$ and dried by nitrogen flow). The deposition chamber was evacuated prior the growth by turbomolecular pump down to the residual pressure $1 \mathrm{mPa}$. The sputtering was performed by argon (purity 99.999\%) at the pressure $1 \mathrm{~Pa}$ and the flow rate $2.0 \mathrm{sccm}$. DC sputtering was performed with the voltage $500 \mathrm{~V}$ and direct current of $0.14 \mathrm{~A}$ for $10 \mathrm{~min}$.

* Institute of Chemistry SAS, Bratislava, Slovakia, chemjumi@savba.sk, ** Institute of Physics CAS, Prague, Czech Republic, *** Faculty of Biomedical Engineering CTU in Prague, Kladno, Czech Republic 


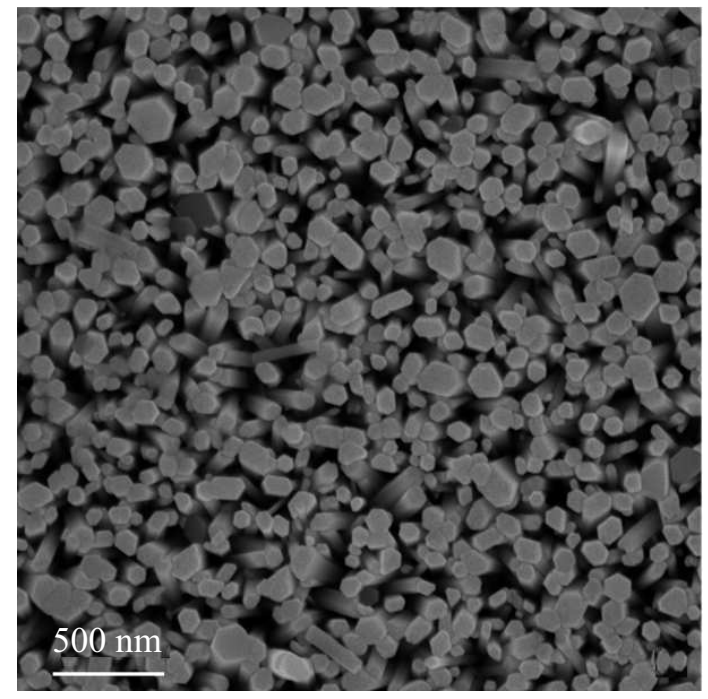

Fig. 1. The SEM image of the top view of $\mathrm{ZnO}$ nanowires

The $\mathrm{ZnO}$ nanowires were prepared by hydrothermal method. The growth solution was prepared from equimolar $(2.5 \mathrm{mmol})$ aqueous solutions of zinc nitrate hexahydrate and hexamethylenetetramine. The mix was blended at room temperature for $30 \mathrm{~min}$ at $350 \mathrm{rpm}$. The growth chamber was filled fresh prepared mixture. The patterned $\mathrm{ZnO}: \mathrm{Al}$ substrates were immersed in the growth solution and then solution was heated at $90{ }^{\circ} \mathrm{C}$ for $3 \mathrm{~h}$. After this time growth of $\mathrm{ZnO}$ nanowires was finished. Finally, the residuals from the surface nanowires were rinsed deionized water and dried by nitrogen flow [22].

The substrates with grown $\mathrm{ZnO}$ nanowires were used to preparing the aqueous colloid by sonication. Ultrasonic treatment was realized by commercial bath sonicator (Elmasonic P - ultrasonic power effective $120 \mathrm{~W}$ ) at frequency $37 \mathrm{kHz}$ for $30 \mathrm{~min}$. The powder of zinc oxide nanowires with defined morphology was produced lyophilization of colloid.

The morphology of zinc oxide nanowires on substrates and after ultrasound peeling was monitored using scanning electron microscopy (SEM) (MAIA3, TESCAN) at voltage $510 \mathrm{keV}$ with InLens detector. The spin coating technique (at $2000 \mathrm{rpm}$ for $1 \mathrm{~min}$ ) and gold mirrors as pads were used for preparation of SEM samples (gold mirrors were treated by oxygen plasma $(100 \mathrm{~W} / \mathrm{min})$ to increase the hydrophilicity of surface for better sticky to nanowires). Dynamic light scattering (DLS) was used for the evaluation of the particle size distribution of colloids. DLS measurements were performed with a Zetasizer Nano ZS (Malvern Instruments Ltd., GB) with detector in position $173^{\circ} \mathrm{C}$ on Backscatter detection. Each sample of suspension $\mathrm{ZnO}$ nanostructures was sonicated for 5 minutes before measurements. Size measurements were performed at $25^{\circ} \mathrm{CC}$ and using clear disposable folded capillary cell (DTS1070).

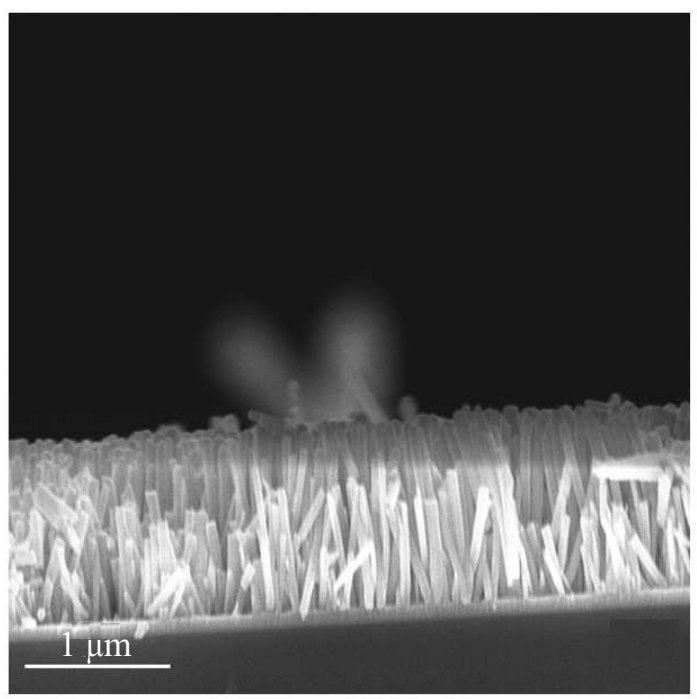

Fig. 2. The SEM image of the cross - sectional view of grown $\mathrm{ZnO}$ nanowires

\section{Experimental results and discussion}

The $\mathrm{ZnO}$ nanowires were synthesized through hydrothermal growth method. It was necessary to use a seeding layer in order to achieve the high quality arrays of single crystalline $\mathrm{ZnO}$ nanowires oriented perpendicularly to the substrate. This layer was created by magnetron sputtering with aluminum-doped zinc oxide ( $\mathrm{ZnO}: \mathrm{Al})$ target. The quality seeding layer was controlled by measure electrical resistivity. The thickness of nucleation layer was monitored and measured by SEM cross-sectional view. And the values thicknesses of individual layers were determinate about $200 \mathrm{~nm}$, see Fig. 2. The preparation of nanostructres was carried out using thermal decomposition zinc nitrate hexahydrate in presence hexamethylenetetramine (HMTA) in aqueous environment. The reaction mixture contained in equimolar (2.5 mmol) ratio both components. The growth synthesis took 3 hours at $90^{\circ} \mathrm{C}$. The morphology (length and diameter) of the nanostructures was characterized using scanning electron microscopy (SEM). Figure 1 demonstrates a typical hexagonal structure of grown nanowires, the average diameter of $\mathrm{ZnO}$ is about $100 \mathrm{~nm}$. Figure 2 represent SEM cross-sectional view, where the average length of nanostructurs is identified about $600 \mathrm{~nm}$.

The powder of $\mathrm{ZnO}$ nanowires was obtained by ultrasound peeling process. At first grown aligned nanostructures were transformed from substrates to colloid in water. Figure 3 illustrate SEM image of sonication process at ultrasonic power $120 \mathrm{~W}$, frequency $37 \mathrm{kHz}$ for $10 \mathrm{~min}$. Finally, obtained colloid was lyophilized. Figure 4 shows SEM image of separated $\mathrm{ZnO}$ nanowires from powder.

Dynamic light scattering (DLS) technique for monitoring of peeling process has proven as unsuitable. The values of average size nanostructures are influenced by the non-spherical shape of $\mathrm{ZnO}$ wires. On the other hand, the scanning electron microscopy (SEM) correctly reflects 


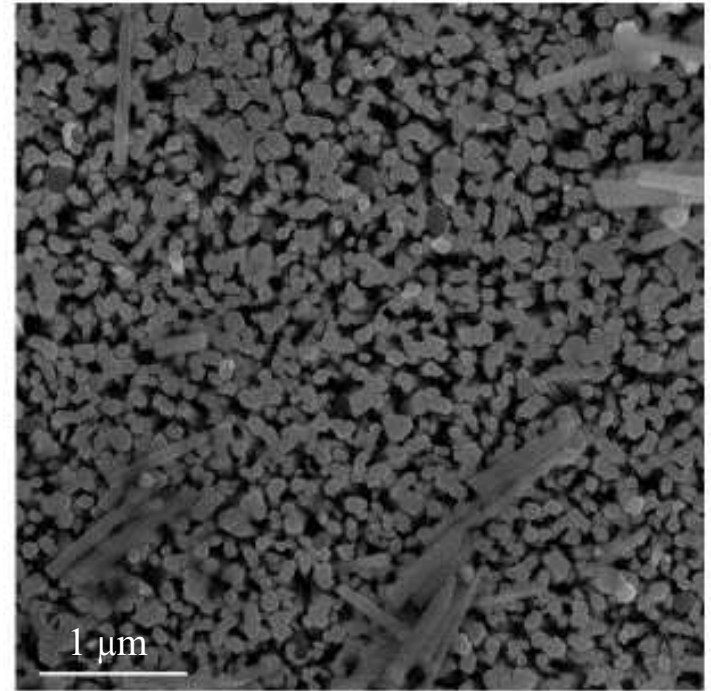

Fig. 3. The SEM image of the top view of $\mathrm{ZnO}$ nanowires on substrate after 10 min sonication

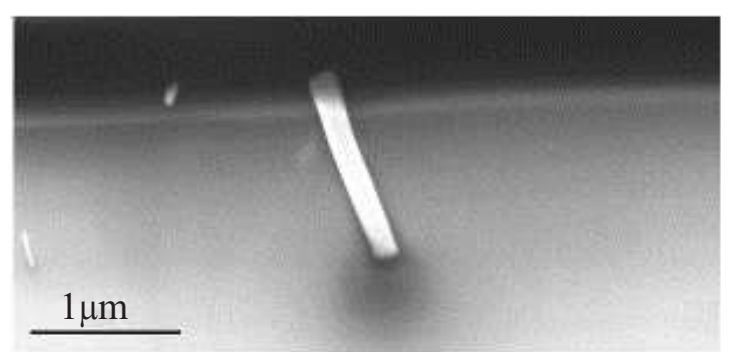

Fig. 4. The SEM image of separated nanowires from $\mathrm{ZnO}$ powder

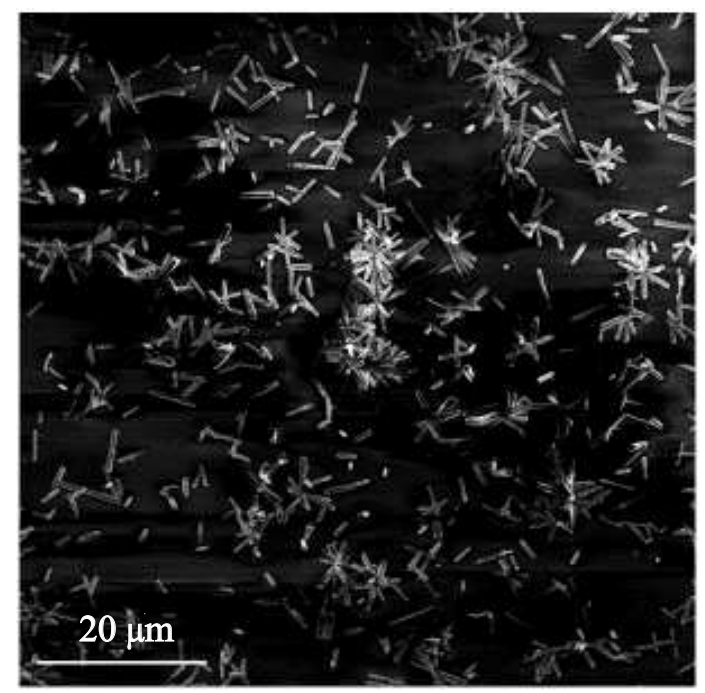

Fig. 5. The SEM image flower like $\mathrm{ZnO}$ nanostructures

the non-spherical shape of nanowires. Thus, only the size values obtained by SEM technique are correct.

Figure 5 shows $\mathrm{ZnO}$ nanostructures in absentia of seeding layer. Figure 6 represents detail form nanostruc-

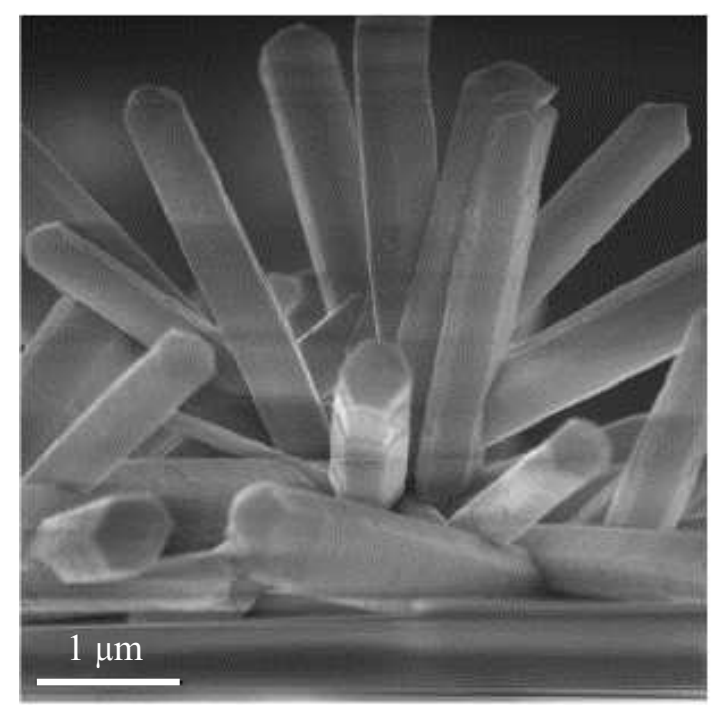

Fig. 6. The SEM image of the detail flower like $\mathrm{ZnO}$

tures and it indicates that a single flower is composed of hexagonal rod-like crystals. Ensues from it that presence the nucleation layer is necessary for production of the nanowires with precisely defined morphology.

\section{Conclusions}

In the present work, we proposed method for mass production powder of zinc oxide nanowires with precisely defined morphology and uniform size distribution. This process is based on thermal growth from zinc nitrate hexahydrate and weak base hexamethylenetetramine (HMTA) in aqueous solution. We demonstrated unavoidable presence the seeding layer during growth process for synthesis arrayed nanowires. The synthesis without the nucleation layer leads to production of flower like $\mathrm{ZnO}$ nanorods. The quality of morphology of produced $\mathrm{ZnO}$ nanowires was investigated by scanning electron microscopy (SEM). SEM technique was used for monitoring peeling process by ultrasound, too. During this process step we obtained colloid of $\mathrm{ZnO}$ nanowires in water, which was subsequently lyophilized to powder.

The proposed approach is simple, cost effective, energy efficient (process at low temperature and atmospheric pressure) and environmentally friendly for synthesis interesting versatile functional material zinc oxide nanowires.

\section{Acknowledgements}

We acknowledge the project NANOCELL KONNECT 007 of Slovak Academy of Sciences and Czech Academy of Sciences the CSF project 16-10429J. Special thanks to Ing. Rayisa Yatskiv from the Institute of Physics of the Czech Academy of Sciences for scanning electron microscopy. 


\section{REFERENCES}

[1] A. Janotti and C. G. Van-de-walle, "Fundamentals of zinc oxide as a semiconductor", Reports on Progress Physics vol. 72 no. 12 (2009), 126501.

[2] M. D. McCluskey and S. J. Jokela, "Defects ZnO", Journal of Applied Physics vol. 106 no. 7 (2009), 71101.

[3] D. Costenaro, F. Carniato, G. Gatti, L. Marchese and C. Bisio, "Preparation of luminescent $\mathrm{ZnO}$ nanoparticles modified with aminopropyltriethoxy silane for optoelectronic applications", New Journal of Chemistr vol. 37, no. 7 (2013), 2103.

[4] J. Mawyin et al "Hybrid Heterojunction Nanorods for Nanoscale Controlled Morphology Bulk Heterojunction Solar Cells", The Journal of Physical Chemistry vol. C 115, no. 21 (2011), 10881-10888.

[5] M. Karimi, J. Saydi, M. Mahmoodi, J. Seidi, M. Ezzati, S. Shamsi Anari and B. Ghasemian, "A comparative study on ethanol gas sensing properties of $\mathrm{ZnO}$ and $\mathrm{Zn0}$. 94Cd0. $06 \mathrm{O}$ nanoparticles", Journal of Physics and Chemistry of Solids vol. 74, no. 10 (2013), 1392-1398.

[6] Ü. Özgür, Y. I. Alivov, C. Liu, A. Teke, M. A. Reshchikov, S. Doğan, V. Avrutin, S. -J. Cho and H. Morkoç "A comprehensive review of $\mathrm{ZnO}$ materials and devices", Journal of Applied Physics, vol. 98 no. 4 (2005), 41301.

[7] T. Frade, M. E. Melo Jorge and A. Gomes, "One-dimensional $\mathrm{ZnO}$ nanostructured films: Effect of oxide nanoparticles", Materials Letters vol. 82 (2012), 13-15.

[8] B. WANG, X. Nikoobakht, A. Herzing and J. Shi, "Scalable synthesis and device integration of self-registered one-dimensional zinc oxide nanostructures and related materials", Chem. Soc. Rev. vol. 42 no. 1 (2013), 342-365.

[9] W. J. Chen, W. L. Liu, S. H. Hsieh and T. K. Tsai, "Preparation of nanosized $\mathrm{ZnO}$ using ? brass", Applied Surface Science vol. 253 no. 16 (2007), 6749-6753.

[10] R. Wahab, S. G. Ansari, Y. -S. Kim, H. -K. Seo and H. -S. Shin, "Room temperature synthesis of needle-shaped $\mathrm{ZnO}$ nanorods via sonochemical method", Applied Surface Science vol. 253 no. 18 (2007), 7622-7626.

[11] Z. W. Pan, "Nanobelts of Semiconducting Oxides", Science vol. 291, no. 5510 (2001), 1947-1949.

[12] W. S. Chiu, P. S. Khiew, M. Cloke, D. Isa, T. K. Tan, S. Radiman, R. Abd-shukor, M. A. A. Hamid, N. M. Huang and H. N. Lim, "Photocatalytic study of two-dimensional $\mathrm{ZnO}$ nanopellets the decomposition of methylene blue", Chemical Engineering Journal vol. 158, no. 2 (2010), 345-352.

[13] V. Polshettiwar, B. Baruwati and R. S. Varma, "Self-Assembly of Metal Oxides into Three-Dimensional Nanostructures", Synthesis and Application Catalysis, ACS Nano vol. 3, no. 3 (2009), 728-736.

[14] Q. Xie, Z. Dai, J. Liang, L. Xu, W. Yu and Y. Qian, "Synthesis of $\mathrm{ZnO}$ three-dimensional architectures and their optical properties", Solid State Communications vol. 136, no. 5 (2005), 304-307.

[15] Y. Zhang, L. Wang, X. Liu, Y. Yan, C. Chen and J. Zhu, "Synthesis of Nano/Micro Zinc Oxide Rods and Arrays by Thermal Evaporation Approach on Cylindrical Shape Substrate", The Journal of Physical Chemistry vol. B 109, no. 27 (2005), 13091-13093.

[16] B. Kotlyarchuk, V. Savchuk and M. Oszwaldowski, "???", Crystal Research and Technology vol. 40, no. 12 (2005), 1118-1123.

[17] J. -J. Wu and S. -C. Liu, "Catalyst-Free Growth and Characterization of ZnO Nanorods", The Journal of Physical Chemistry vol. B 106, no. 37 (2002), 9546-9551.

[18] D. J. Lee, J. Y. Park, Y. S. Yun, Y. S. Hong, J. H. Moon, B. -T. Lee and S. S. Kim, "Comparative studies on the growth behavior of $\mathrm{ZnO}$ nanorods by metalorganic chemical vapor deposition depending on the type of substrates", Journal of Crystal Growth vol. 276, no. 3-4 (2005), 458-464.

19] M. Zheng, L. Zhang, G. Li and W. Shen, "Fabrication and optical properties of large-scale uniform zinc oxide nanowire arrays by one-step electrochemical deposition technique", Chemical Physics Letters vol. 363, no. 1-2 (2002), 123-128.

20] J. Jie, G. Wang, Q. Wang, Y. Chen, X. Han, X. Wang and J. G. Hou, "Synthesis and Characterization of Aligned ZnO Nanorods on Porous Aluminum Oxide Template", The Journal of Physical Chemistry vol. B 108, no. 32 (2004), 11976-11980.

[21] L. E. Greene, M. Law, J. KIM, F. Goldberger, J. C. Johnson, Y. Zhang, R. J. Saykally and P. Yang, "Low-Temperature Wafer-Scale Production of ZnO Nanowire Arrays", Angewandte Chemie International Edition vol 42, no. 26 (2003) 3031-3034.

$22]$ N. Neykova, J. Stuchlik, K. Hruska, A. Poruba, Z. Remes and O. Pop-Georgievski, "Study of the surface properties of $\mathrm{ZnO}$ nanocolumns used for thin-film solar cells", Beilstein Journal of Nanotechnology vol. 8, (2017), 446-451.

Received 23 April 2017

Júlia Mičová, born in Slovakia (1974), graduated from the Faculty of Chemical Technology, STU Bratislava, in 1999, received the $\mathrm{PhD}$ degree in 2005 from Faculty of Natural Sciences, Comenius University in Bratislava. She received a scholarship fund of Štefan Schwarz for postdoctoral position at Slovak Academy of Sciences in 2005. She has worked as a researcher at Institute of Chemistry Slovak Academy of Sciences, Bratislava, Slovakia. Her scientific interest is focused on surface modification of inorganic nanostructures with organic molecules. She co-authored 16 scientific peer-reviewed journal contributions.

Yu-Ying Chang, born in Taiwan, ROC (1988), received his BSc degree in applied physics from National Pintung University of Education, Taiwan (2012) and MSc degree in optoelectronics and material science from National Pintung University of Education, Taiwan (2014). He worked as a research assistant in the field of magnetic circular dichroism at the Department of Applied Physics, National Pintung University, Taiwan (2015-2016) and at the Department of Optical Materials, Institute of Physics of the Czech Academy of Sciences, Prague, Czech Republic (since 2016). He is a PhD student at the Faculty of Biomedical Engineering Czech Technical University in Prague (since 2016). His research interests include deposition of thin films, plasma treatment, surface fictionalization, optical and electrical measurements. He is the co-author of 3 indexed journal papers and 3 conference papers.

Zdeněk Remeš, born in Czech Republic (1971), received his MSc degree in solid state physics (1994) and $\mathrm{PhD}$ degree in solid state physics and material research (1999) from the Faculty of Mathematics and Physics, Charles University, Prague, Czech Republic. His postdoctoral fellow attachments were at the Institute of Materials Research, Hasselt University, Belgium (1999-2002, 2003-2004), the Solid State Institute, Technion - Israel Institute of Technology, Haifa (2002-2003) and CEA/Saclay, France (2006). He works as a researcher at the Department of Optical Materials, Institute of Physics of the Czech Academy of Sciences, Prague, Czech Republic (since 1993) and as the assistant professor at the Faculty of Biomedical Engineering (FBMI) of the Czech Technical University. His research interests include optical and electrical properties of thin films, optical absorption, photocurrent, photoluminescence and infrared spectroscopy. He is the co-author of 106 documents indexed in Scopus (H-factor 20). 